INPLASY

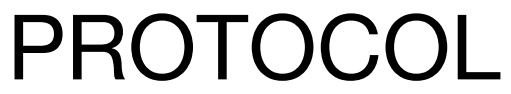

To cite: Brito et al. Simulated

Soccer Games Protocols:

What we Know so far-A

Systematic Review. Inplasy

protocol 202120080. doi:

10.37766/inplasy2021.2.0080

Received: 27 February 2021

Published: 27 February 2021

Corresponding author:

Pedro Brito

pbrito49@gmail.com

Author Affiliation:

Research Center in Sports

Sciences, Health Sciences and

Human Development, CIDESD,

University of Maia, ISMAI,

Maia, Portugal

Support: None.

Review Stage at time of this submission: Formal screening of search results against

eligibility criteria.

Conflicts of interest:

None declared.

\section{Simulated Soccer Games Protocols: What we Know so far-A Systematic Review}

Review question / Objective: The aim of this review is to discuss simulated soccer match protocols analysing their strengths, weaknesses, validity and reliability.

Condition being studied: Fatigue during and after simulated soccer match protocols.

Information sources: Electronic databases (PubMed, SPORTDiscus via EBSCOhost, Web of Science and Scopus) were searched for relevant publications prior to the 31 January 2021.

INPLASY registration number: This protocol was registered with the International Platform of Registered Systematic Review and Meta-Analysis Protocols (INPLASY) on 27 February 2021 and was last updated on 23 August 2021 (registration number INPLASY202120080).

\section{INTRODUCTION}

Review question / Objective: The aim of this review is to discuss simulated soccer match protocols analysing their strengths, weaknesses, validity and reliability.
Rationale: Protocols can be used to simulate physical and physiological demands that occur during a soccer match.

Condition being studied: Fatigue during and after simulated soccer match protocols. 


\section{METHODS}

Search strategy: The searches for relevant content related to fatigue development during simulated-soccer match tests or protocols were performed until the end of January 2021, using four electronic databases: PubMed, SPORTDiscus via EBSCOhost, Web of Science and Scopus. In each database the following descriptors were used: (1) [soccer OR football*] AND (2) [match* OR game* OR competit ${ }^{\star}$ ] (3) [simulat* OR test* OR protocol* OR treadmill] AND (4) [fatigue OR tired* OR exhaustion OR weak*] AND (5) [valid* OR Reliab* OR Reproduc*] and NOT (6) ["American football" OR "Australian football" OR "Beach soccer" OR Indoor OR "Gaelic football" OR Rugby OR Futsal].

Participant or population: Soccer players from any sex, adults.

Intervention: Soccer Players exposed to protocols on fatigue development.

Comparator: Simulated soccer-related matches.

Study designs to be included: Observational studies with a before-after intervention (i.e., simulated-soccer match tests or protocols).

Eligibility criteria: (1) original article, (2) abstract available for screening, (3) published in English language, (4) published/ahead of print up to and including January 2021, (5) in a scientificindexed peer-reviewed journal (i.e., abstracts published in conference proceedings, books, theses, dissertations, reviews, systematic reviews and metaanalyses were not considered), (6) studies conducted in soccer; (7) simulated-soccer match tests or validated simulation protocols (on-field or treadmill), (8) included at least one outcome measure of post-simulated-match test or protocol, such as neuromuscular performance, external load, internal load or psychometric state. No restrictions regarding the date of publication were imposed other than those described in item 4. (8) No sex restriction was applied and age > 18 (i.e., adults) was considered, (9) studies reporting descriptive effects of soccer fatigue protocols on fatigue (validation studies).

Information sources: Electronic databases (PubMed, SPORTDiscus via EBSCOhost, Web of Science and Scopus) were searched for relevant publications prior to the 31 January 2021.

Main outcome(s): Dependent variables are similar changes in biochemical, neuromuscular, and perpetual measures compared to changes found in soccer matches.

Quality assessment / Risk of bias analysis: National Heart Blood Institute (NIH) guidelines for qualitative evaluation of observational cohort and cross-sectional studies were considered. The quality assessment was based on the reporting of study methods and results with answer categories being "yes," "partial," and "no"). Summary scores (ranging from 0 to 1) were calculated as follows: Summary score = [(number of 'yes' $\times 2$ ) + (number of 'partial' $\times 1$ )] / (number of criteria $\times 2$ ) (1) Studies were then classified as high $(\geq 0.75)$, moderate $(0.50-0.75)$, or poor methodological quality $(<0.50)$. The revised Risk of Bias Assessment Tool for Nonrandomized Studies (RoBANS) was used to judge the risk of bias at study-level. RoBANS contains six domains: the selection of participants, confounding variables, the measurement of exposure, the blinding of the outcome assessments, incomplete outcome data, and selective outcome reporting, and each study was classified as Low Risk, Unclear and High Risk.

Strategy of data synthesis: None.

Subgroup analysis: None.

Sensitivity analysis: None.

Language: English.

Country(ies) involved: Portugal. 
Keywords: Soccer; Simulated Protocols; Fatigue; Physiology.

Contributions of each author:

Author 1 - Pedro Brito - Conceptualization, Formal analysis, Investigation, Methodology, Run the data search, analyzed and interpreted the data, Writing original draft, Writing - review \& editing.

Email: pbrito49@gmail.com

Author 2 - Júlio Costa - Conceptualization, Formal analysis, Investigation, Methodology, Analyzed and interpreted the data, Writing - original draft, Writing review \& editing.

Email: jahdc@hotmail.com

Author 3 - Pedro Figueiredo - Methodology, Supervision, Writing - review \& editing.

Email: pedfig@me.com

Author 4 - João Brito - Methodology, Project administration, Supervision, Writing

- review \& editing.

Email: joao.brito@fpf.pt 